\title{
AVALIAÇÃO DA EFETIVIDADE E SEGURANÇA DE NOVOS ANTICOAGULANTES ORAIS COMPARADOS À ENOXAPARINA NA PREVENÇÃO DO TROMBOEMBOLISMO VENOSO EM PACIENTES SUBMETIDOS À CIRURGIA ELETIVA DE ARTROPLASTIA DE JOELHO E QUADRIL
}

\author{
L. A. OLIVEIRA ${ }^{1}$, F. S. ROCHA ${ }^{1}$, M, D.L. OLIVEIRA ${ }^{1}$, \\ F. ZANGHELINI ${ }^{1}$, C.A.S. ANDRADE ${ }^{1}$ \\ ${ }^{1}$ Universidade Federal de Pernambuco, Centro de Ciências Biológicas, Departamento de \\ Bioquímica \\ E-mail para contato: lucianaalcoforado@gmail.com

\begin{abstract}
RESUMO - Objetivo: avaliar a efetividade e segurança dos novos anticoagulantes orais (NACOs) apixabana, edoxabana, rivaroxabana (inibidores do Fator Xa) e Dabigatrana (Inibidor direto da trombina), intervenção, quando comparados à enoxaparina, controle, na prevenção do tromboembolismo venoso em pacientes adultos submetidos à cirurgia eletiva de artroplastia de joelho ou de quadril. Metodologia: revisão sistemática com metanálise de ensaios clínicos randomizados pesquisados nas bases de dados MEDLINE (via Pubmed), Center for Reviews and Dissemination (CRD) e Biblioteca COCHRANE, e pesquisa na literatura cinza. Os estudos avaliados apontam para a não inferioridade dos NACOs em relação à enoxaparina. As metanálises indicam que não há diferença estatística significativa entre o grupo intervenção e o grupo controle, para o desfecho sangramento não-maior clinicamente relevante.
\end{abstract}

Palavras-chave: apixabana, dabigatrana, edoxabana, rivoraxabana, tromboembolismo venoso, artroplastia de quadril, artroplastia de joelho.

ABSTRACT - Objective: evaluate the effectiveness and safety of new oral anticoagulants (NACOs) apixaban, edoxaban, rivaroxaban (Factor Xa inhibitors) and dabigatran (direct thrombin inhibitor), intervention, when compared to enoxaparin, control, in the prevention of venous thromboembolism in adult patients undergoing elective surgery of knee or hip arthroplasty. METHODS: Systematic review with a meta-analysis of randomized clinical trials searched in MEDLINE (via Pubmed), Center for Reviews and Dissemination (CRD) and COCHRANE Library, and research in the gray literature. The evaluated studies indicate a non-inferiority of NACOs in relation to enoxaparin. The meta-analyzes indicate a statistically non-significant difference between the intervention group and the control group for the clinically relevant non-major bleeding.

Keywords: apixaban, dabigatran, edoxabana, rivoraxabana, venous thromboembolism, hip arthroplasty, knee arthroplasty. 


\section{INTRODUÇÃO}

Rivaroxabana, dabigatrana, apixabana e edoxabana são os quatro novos anticoagulantes orais disponíveis (NACOs) que estão atualmente aprovados para profilaxia do tromboembolismo venoso após a reposição total do quadril e do joelho. Estudos de fase 3 e fase 4 que comparam o NOAC com heparinas de baixo peso molecular mostraram resultados similares quanto à eficácia e segurança dessas duas categorias de anticoagulantes. Entre eles, há a comparação entre os inibidores do Fator $\mathrm{Xa}$ e os inibidores diretos da trombina (NACOs) com enoxaparina e seu uso em cirurgias ortopédicas da artroplastia do joelho ou do quadril, que serão objetos do presente estudo.

Os estudos coletados em revisão sistemática, foram submetidos à meta-análise para avaliar a eficácia e segurança do seu uso dos NACOs para prevenir tromboembolismo venoso em pacientes submetidos a cirurgia de artroplastia de joelho ou quadril, estudos como este facilitarão a escolha dos profissionais de saúde no momento da tomada de decisões.

\section{MATERIAIS E MÉTODOS}

Foi utilizado o DeCS da Biblioteca Virtual em Saúde e o MeSH da Medline Pubmed para obtenção dos descritores (palavras-chave). Montou-se uma estratégia de busca predefinida para os seguintes bancos de dados: MEDLINE (via Pubmed), Center for Reviews and Dissemination (CRD), LILACS e Biblioteca COCHRANE, também foi pesquisada a literatura cinzenta. Foram consideraram publicações nas línguas inglesa, espanhola e portuguesa. Ao final da busca, quatro artigos foram selecionados.

Pergunta de pesquisa: O uso dos NACOs é efetivo e seguro na prevenção do tromboembolismo venoso em pacientes adultos submetidos adultos submetidos à cirurgia eletiva de artroplastia de joelho ou de quadril, quando comparado ao uso da enoxaparina?

O desfecho primário sobre a análise de efetividade e segurança do uso dos NACOs será associada a prevenção do tromboembolismo venoso (TEV) em pacientes adultos submetidos à cirurgia eletiva de artroplastia de joelho ou quadril. Serão analisados os desfechos: $01=$ Embolia pulmonar; $02=$ Trombose venosa profunda sintomática; $03=$ Trombose venosa profunda assintomática; $04=$ Sangramento maior; $05=$ Sangramento não-maior clinicamente relevante.

Critérios de inclusão: (1) Revisões sistemáticas (com ou sem meta-análise), bem como ensaios clínicos randomizados não cobertos por análises sistemáticas; (2) População: pacientes adultos submetidos a cirurgia eletiva de artroplastia de joelho ou de quadril usando anticoagulante para prevenir tromboembolismo venoso (TEV); (3) Estudos cujo desenho de avaliação incluía apixabana, edoxabana, dabigatrana e rivaroxabana no grupo de intervenção e a enoxaparina no grupo comparador; (4) Avaliado como resultado, segurança e eficácia terapêutica, incluindo um ou mais desfechos, tais como EP, TVP sintomática, TVP assintomática, sangramento maior; sangramento não maior clinicamente relevante. E critérios de exclusão: (1) estudos comparativos não randomizados ou revisões narrativas, (2) estudos publicados em outras línguas que não o inglês, espanhol e português, (3) uso dos NACOs combinados com outras alternativas terapêuticas no grupo de controle (4), dados incompletos, e (5) que não avaliou os resultados em um ou mais dos desfechos abordados. 


\section{RESULTADOS E DISCUSSÃO}

Os dados de ensaios clínicos para os novos anticoagulantes orais demonstram efetividade e segurança comparáveis às da enoxaparina. No entanto, as vantagens desta nova geração de medicamentos, incluindo a administração oral, a dosagem fixa, as janelas terapêuticas amplas e a necessidade de monitorização rotineira da coagulação, sugerem que esses agentes podem ser alternativas para pacientes submetidos à cirurgia ortopédica maior que correm o risco de TEV.

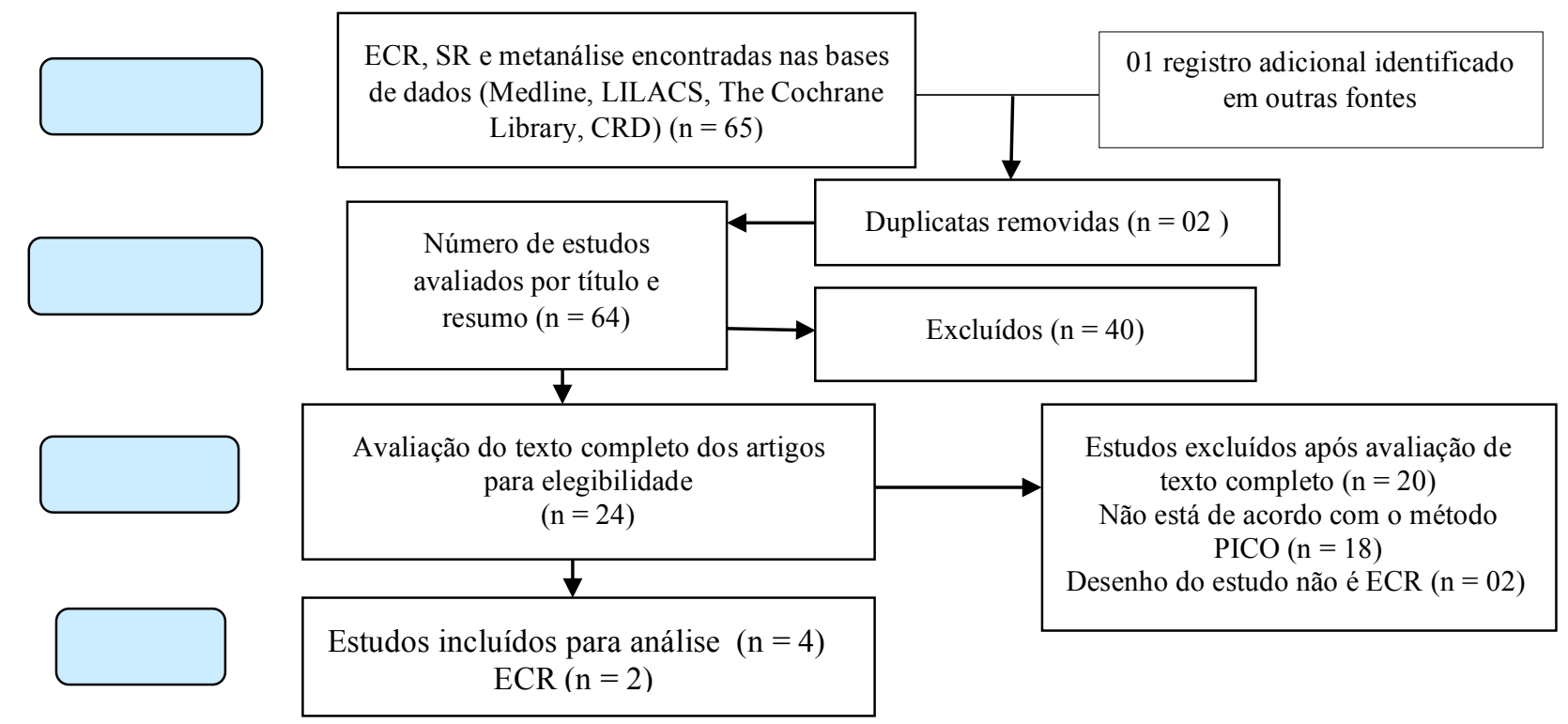

Avaliação da qualidade metodológica

\begin{tabular}{|c|c|c|c|c|c|c|c|c|c|c|c|}
\hline Artigo & 1 & 2 & 3 & 4 & 5 & 6 & 7 & 8 & 9 & 10 & Score \\
\hline $\begin{array}{l}\text { Forster R, Stewart } \\
\text { M., } 2016\end{array}$ & SIM & SIM & SIM & SIM & SIM & SIM & SIM & SIM & SIM & SIM & $10 / 10$ \\
\hline $\begin{array}{l}\text { Salazar CA, } \\
\text { Malaga G, } \\
\text { Malasquez G., } \\
2011\end{array}$ & SIM & SIM & SIM & SIM & SIM & SIM & SIM & SIM & SIM & SIM & $10 / 10$ \\
\hline $\begin{array}{l}\text { Mahan CE, Kaatz } \\
\text { S., } 2012\end{array}$ & SIM & SIM & SIM & SIM & SIM & SIM & SIM & $\begin{array}{c}\mathrm{NA} \\
\mathrm{O}\end{array}$ & $\begin{array}{c}\mathrm{NA} \\
\mathrm{O}\end{array}$ & $\begin{array}{c}\mathrm{NA} \\
\mathrm{O}\end{array}$ & $7 / 10$ \\
\hline $\begin{array}{l}\text { Fuji, Takeshi et } \\
\text { al., } 2014\end{array}$ & $\begin{array}{c}\mathrm{NA} \\
\mathrm{O}\end{array}$ & SIM & SIM & SIM & SIM & SIM & SIM & SIM & $\begin{array}{c}\mathrm{NA} \\
\mathrm{O}\end{array}$ & SIM & $8 / 10$ \\
\hline
\end{tabular}

$1=$ Um projeto anterior foi fornecido?

$2=$ Foi realizada uma pesquisa bibliográfica abrangente?

$3=$ O status da publicação foi utilizado como critério de inclusão?;

4 = Uma lista de estudos (incluídos e excluídos) foi fornecida?;

5 = As características dos estudos incluídos foram fornecidas?;

6 = A qualidade científica dos estudos incluídos foi avaliada e documentada?

7 = A qualidade científica dos estudos incluídos foi adequadamente utilizada na formulação das conclusões?

8 = Os métodos usados para combinar os resultados de estudos foram apropriados?

$09=$ A probabilidade de viés de publicação foi avaliada?

$10=\mathrm{O}$ conflito de interesses foi informado? 
Desfecho: Sangramento não-maior clinicamente relevante

\begin{tabular}{|c|c|c|c|c|c|}
\hline Stutruor Subaroun & $\begin{array}{l}\text { Intervent } \\
\text { Events }\end{array}$ & Ition Compari & rison & $\begin{array}{c}\text { Odds Ratio } \\
\text { M.H Random }\end{array}$ & $\begin{array}{c}\text { Odds Ratio } \\
\text { M.H Random } 95 \% \text { Cl }\end{array}$ \\
\hline Forster R, Stewart M., 2016 & 300 & 8341 & $715594.9 \%$ & $1.09[0.92,1.30]$ & \\
\hline Fuji, Takeshi etal., 2014 & 18 & 354 & $5.1 \%$ & $1.50[0.71,3.17]$ & \\
\hline Total (95\% Cl) & & 8695 & $7504100.0 \%$ & $1.11[0.94,1.32]$ & 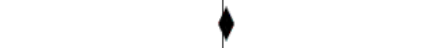 \\
\hline Total events & 318 & 248 & & & \\
\hline \multicolumn{5}{|c|}{$\begin{array}{l}\text { Heterogeneity: Tau } \|^{2}=0.00 ;\left.C h\right|^{2}=0.67, d f=1(P=0.41) ; 1^{2}=0 \% \\
\text { Test for overall effect: } Z=1.23(P=0.22)\end{array}$} & 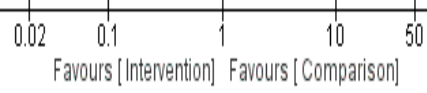 \\
\hline
\end{tabular}

\section{CONCLUSÃO}

Para o desfecho sangramento não-maior clinicamente relevante, os resultados obtidos não indicam diferença estatística significativa entre o grupo intervenção e o grupo controle.

\section{REFERÊNCIAS}

- FORSTER R, STEWART M. Anticoagulants (extended duration) for prevention of venous thromboembolism following total hip or knee replacement or hip fracture repair. Cochrane Database Syst Rev 2016;3:CD004179-CD004179.

- FUJI T, WANG CJ, FUJITA S, KAWAI Y, NAKAMURA M, KIMURA T ET AL. Safety and efficacy of edoxaban, an oral factor Xa inhibitor, versus enoxaparin for thromboprophylaxis after total knee arthroplasty: the STARS E-3 trial. Thromb Res 2014;134:1198-204.

- MAHAN CE, KAATZ S. Performance of new anticoagulants for thromboprophylaxis in patients undergoing hip and knee replacement surgery. Pharmacotherapy. 2012; 32(11):1036-1048. doi:10.1002/phar.1133.

- SALAZAR CA, MALAGA G, MALASQUEZ G. Direct thrombin inhibitors versus vitamin $\mathrm{K}$ antagonists or low molecular weight heparins for prevention of venous thromboembolism following total hip or knee replacement. Cochrane Database of Systematic Reviews 2010, Issue 4. Art. No.: CD005981. DOI: 10.1002/14651858.CD005981.pub2. 